\title{
Improvement of Software for Dynamic Force Correction of Force Transducers
}

\author{
Naoki Miyashita ${ }^{a,}{ }^{,}$, Ryosuke Araki ${ }^{a}$, Akihiro Takita $^{a}$, Takao Yamaguchi $^{\mathrm{a}}$, Agus Setyo Budi $^{\mathrm{b}}$, Yusaku Fujii ${ }^{\mathrm{a}}$

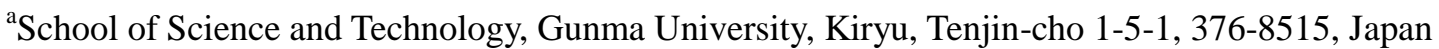 \\ ${ }^{\mathrm{b}}$ Universitas Negeri Jakarta, JI. Pemuda 10, Rawamangun Jakarta 13220 Indonesia \\ *Corresponding Author: t10306070@gunma-u.ac.jp
}

\begin{abstract}
Software for correcting the dynamic error of the force sensor in the impact force measurement, which uses an output signal of the sensor itself, has been improved. The software corrects the output waveform of the sensor by using the output waveform itself and estimates its uncertainty. In the experiment, the dynamic error of the sensor is evaluated using the Levitation Mass Method (LMM). In the LMM, the impact force applied to the sensor is accurately determined as an inertial force of the moving part of the aerostatic linear bearing. The parameter for correcting the dynamic error is determined from the results of one set of impact measurements of the sensor. Then the validity of the parameter is evaluated by using the results of measurements of the sensor. The uncertainty of the un-corrected force and that of the corrected force are also estimated. In the previous version of the software, the user input "input text-file" which contains force that is calculated based on the static calibration results. On the other hand, the output signal from the sensor is not force. This time, the software has been modified to load input text-file which contains the output signal from the sensor.
\end{abstract}

Keywords: Inertial force, Dynamic calibration, Levitation Mass Method.

\section{Introduction}

Force sensors are used in both industrial and research areas. However, only standard calibration methods of static force are available at present. This fact results in that it is difficult for users of the sensors to estimate dynamic error and to correct the error.

A method of the Levitation Mass Method (LMM) has been proposed for calibration of the dynamic forces ${ }^{(1)}$. In the LMM, varying force such as an impact force is applied to the sensor. The force is regarded as inertial force of a mass supported using an aerostatic linear bearing, i.e., moving part of the bearing. In the method, only Doppler frequency shift of laser light reflected by the mass is measured using an optical interferometer. Thereafter, other quantities such as velocity, acceleration, displacement and inertial force are calculated from the Doppler frequency shift.

Fujii et al. showed that the error in impact force measurement is approximately proportional to the acceleration at detection point of an S-shaped strain-gage force sensor, and further, it can be described as an effect of the inertial force of a portion of the sensor itself. The difference between the static and dynamic characteristics of the sensor is well explained as the inertial force of a portion of the sensor itself ${ }^{(2)}$. This result suggests that it is possible to correct the dynamic measurement error by measuring the acceleration of the detection point.

The dynamic error of the force sensor, which could be corrected by using the output signal of their own, is shown ${ }^{(3)}$. It is confirmed that the acceleration at the detection point of the sensor is roughly proportional to the differential coefficient of the second order of the output signal of the sensor itself.

On the basis of the method proposed above, software which corrects the dynamic error using the output signal of the force sensor itself has been developed ${ }^{(4)}$. This software needs to be input "input text-file" which contains force that is calculated based on the static calibration results. However, the output signal from the sensor is not force. So, we develop software which is input "input text-file" which contains the output signal from the sensor. This 
improvement enhances convenience of the software.

In this paper, we evaluate experimentally effectiveness of the proposed method to correct the dynamic error and to evaluate the uncertainty. Then, we describe the effectiveness of the software which is modified to input the output signal from the sensor.

\section{Experiment}

Figure 1 shows a schematic diagram of experimental setup for measuring the impact response of a force sensor. The sensor under test is fixed to a large metal base. An aerostatic linear bearing is used to obtain a linear motion with small friction enough to act on the mass, i.e., the moving part of the bearing. The initial velocity is applied to the moving part by hand.

The velocity of the mass $v_{1}$ is measured using an optical interferometer. Force acting on the detection point of the sensor is obtained by the equation of motion, $\boldsymbol{F}=\boldsymbol{m a}$, where $\boldsymbol{m}$ is the mass and $\boldsymbol{a}$ is the acceleration.

The mass of the moving part which contains the cube corner prism (CC) and the extension $\operatorname{rod}, \boldsymbol{M}$, is approximately $2.653 \mathrm{~kg}$.

A conventional S-shaped strain-gage type force sensor (model: DB-200N, capacity: 200N manufacture: Showa Measuring Instruments Co., Ltd., Tokyo, Japan) is used in the experiment. The manufacture year of the sensor is 2008 . This sensor's static calibration coefficient, $\boldsymbol{C}_{\mathbf{s}}$, is 66.8 $\mathrm{N} / \mathrm{mV} / \mathrm{V}$.

A Zeeman-type two-frequency He-Ne laser is used as the light source of the optical interferometer. The interferometer has two photo-detectors: PD0 and PD1. The frequency difference between the orthogonal polarization states of the two outputs from the laser, $f_{\text {rest }}$, is monitored with a Glan-Thompson prism (GTP) and PD0.

The total force acting on the moving part of the aerostatic linear bearing, $\boldsymbol{F}_{\text {mass }}$, is calculated as the product of the mass, $\boldsymbol{M}$, and the acceleration, $\boldsymbol{a}$, as follows:

$$
\boldsymbol{F}_{\text {mass }}=\boldsymbol{M a}
$$

In the experiment, friction force acting on the mass is negligible because it is sufficiently small, the total force acting on the mass, $\boldsymbol{F}_{\text {mass }}$, is same as force acting on the mass of the sensor to be tested ${ }^{(5)}$. The acceleration is calculated from the velocity of the floating mass. The velocity of the mass, i.e., moving part of the aerostatic linear bearing, $v$, is measured as the Doppler shift frequency, $f_{\text {Doppler, }}$, which is expressed as follows:

$$
\begin{aligned}
& v=\lambda_{\text {air }}\left(f_{\text {Doppler }}\right) / 2 \\
& f_{\text {Doppler }}=-\left(f_{\text {beat }}-f_{\text {rest }}\right)
\end{aligned}
$$

where $\lambda_{\text {air }}$ is wavelength of the signal beam under the experimental conditions and $\boldsymbol{f}_{\text {beat }}$ is beat frequency, which is frequency difference between signal beam and reference

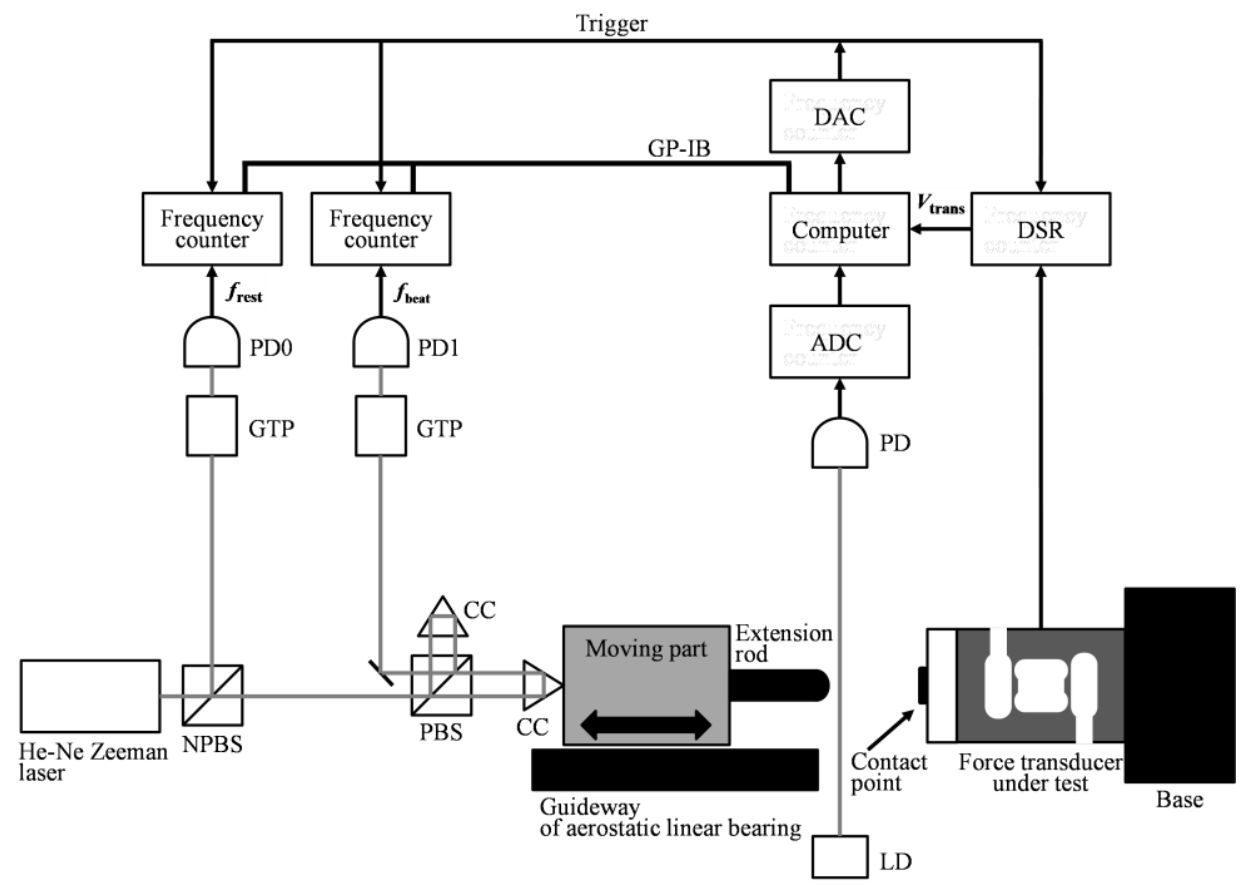

Fig. 1. Experimental setup. Code: PBS $=$ Polarizing beam splitter, NPBS $=$ Non-polarizing beam splitter, $\mathrm{CC}=\mathrm{Cube}$ corner prism, GTP = Glan-Thompson prism, $\mathrm{PD}=$ Photo detector, $\mathrm{LD}=$ Laser diode, $\mathrm{ADC}=$ Analog-to-digital converter, DAC $=$ Digital-to-analog converter, DSR $=$ Dynamic strain recorder 
beam, appears at PD1. The position of the mass, $\boldsymbol{x}$, the acceleration of the mass, $\boldsymbol{a}$, and the force acting on the mass, $\boldsymbol{F}_{\text {mass }}$, are calculated from the velocity, $\boldsymbol{v}$.

For simultaneous measurement, two frequency counters and the dynamic strain recorder (DSR) are triggered by a single signal originated by a light switch made of a laser diode (LD) and a PD.

The output signal of the sensor, $\boldsymbol{V}_{\text {trans, }}$ is stored by a dynamic strain recorder (DSR; model: DC-204R, Tokyo Sokki Kenkyujo, Tokyo, Japan) and done unit conversion from strain to $\mathrm{mV} / \mathrm{V}$ from the relationship of $2000 \times 10^{-6}$ strain $=1 \mathrm{mV} / \mathrm{V}$. " $\mathrm{mV} / \mathrm{V}$ " is used for indication of rated output of the sensor and shows the output voltage $(\mathrm{mV})$ for bridge voltage $1 \mathrm{~V}$. The force measured by the sensor, $\boldsymbol{F}_{\text {trans, }}$, is calculated using the output signal of the sensor, $\boldsymbol{V}_{\text {trans, }}$ and its static calibration result. The force calculated using the output signal of the sensor, $\boldsymbol{V}_{\text {trans, and its static }}$ calibration result, $\boldsymbol{F}_{\text {trans, }}$, is compared with the measured force as the inertial force of the mass, $\boldsymbol{F}_{\text {mass }}$.

In the experiment, one set of experiment consists of 20 impact measurements. First, the experiment is conducted for evaluating parameter for dynamic error correction. Then, validity of the estimated parameter is evaluated.

\section{Estimation and Evaluation of the Parameters for Dynamic Error Correction}

Figure 2 and Figure 3 show result of a single set of the impact test of the force sensor. Figure 2 shows the output signal of the sensor, $\boldsymbol{V}_{\text {trans }}$.

The force measured by the sensor, $\boldsymbol{F}_{\text {trans, }}$, is calculated as product of its output signal, $\boldsymbol{V}_{\text {trans, and its static }}$ calibration coefficient, $\boldsymbol{C}_{\mathbf{s}}$.

Figure 3 shows the force measured by the sensor, $\boldsymbol{F}_{\text {trans, }}$, and the force acting on the mass, $\boldsymbol{F}_{\text {mass, }}$, and their

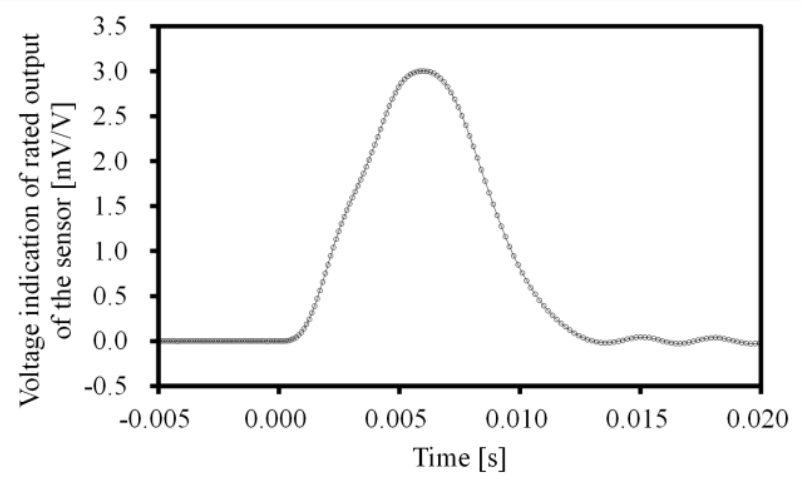

Fig. 2. The output signal of the force sensor, $\boldsymbol{V}_{\text {trans }}$.

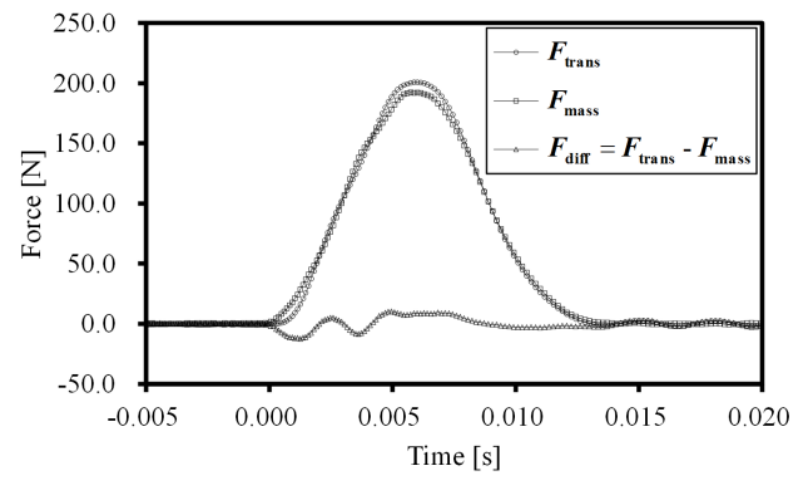

Fig. 3. The force measured by the sensor, $\boldsymbol{F}_{\text {trans, }}$, the force acting on the mass, $\boldsymbol{F}_{\text {mass, }}$ and the difference between the forces, $\boldsymbol{F}_{\text {diff. }}$

difference, $\boldsymbol{F}_{\text {diff: }}$

$$
\boldsymbol{F}_{\text {diff }}=\boldsymbol{F}_{\text {trans }}-\boldsymbol{F}_{\text {mass }}
$$

The force measured by the sensor, $\boldsymbol{F}_{\text {trans }}$, is obtained by the method described above. The difference between $\boldsymbol{F}_{\text {trans }}$ and $\boldsymbol{F}_{\text {mass }}$ is derived from the difference between the static and dynamic characteristics of the sensor mainly.

Figure 4 shows relationship between the second-order time derivatives of the force measured using the sensor, $\mathrm{d}^{2} \boldsymbol{F}_{\text {trans }} / \mathrm{d} \boldsymbol{t}^{2}$, and $\boldsymbol{F}_{\text {diff. }}$

The plots show a linear relationship between $\mathrm{d}^{2} \boldsymbol{F}_{\text {trans }} /$

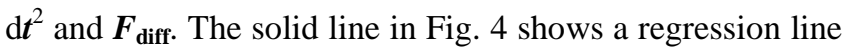
in the following equation:

$$
\boldsymbol{F}_{\text {reg }}=\boldsymbol{C}_{\mathrm{d}}\left(\mathrm{d}^{2} \boldsymbol{F}_{\text {trans }} / \mathrm{d} \boldsymbol{t}^{2}\right)
$$

where $\boldsymbol{C}_{\mathbf{d}}$ is the parameter for dynamic error estimation.

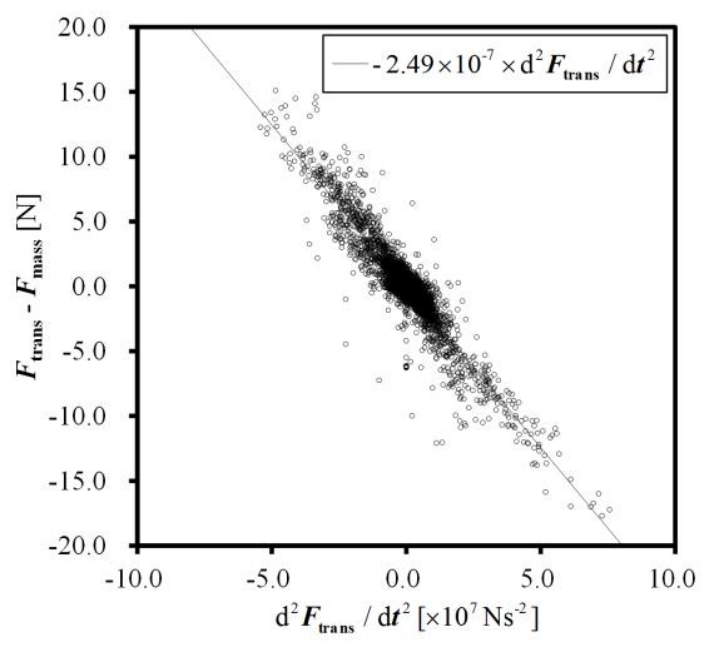

Fig. 4. Relationship between the second-order time derivatives of the force measured using the sensor, $\mathrm{d}^{2} \boldsymbol{F}_{\text {trans }} / \mathrm{d} \boldsymbol{t}^{2}$, and $\boldsymbol{F}_{\text {diff- }}$ 


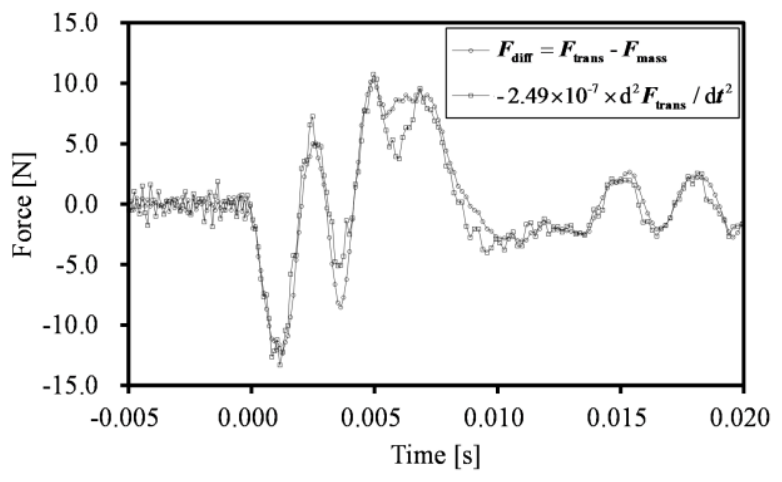

Fig. 5. Difference between $\boldsymbol{F}_{\text {trans }}$ and $\boldsymbol{F}_{\text {mass }}$ and inertial force of the sensor.

The parameter for dynamic error estimation, $\boldsymbol{C}_{\mathbf{d}}$, is obtained as slope of the regression line. From the Fig. 4, the regression line is determined by a least-square method and $\boldsymbol{C}_{\mathbf{d}}=-2.49 \times 10^{-7} \mathrm{~s}^{2}$ is obtained. The measured plots are in good agreement with the regression line.

Figure 5 shows the difference between $\boldsymbol{F}_{\text {trans }}$ and $\boldsymbol{F}_{\text {mass }}$ and inertial force of the sensor which is estimated by the parameter, $\boldsymbol{C}_{\mathbf{d}}, \boldsymbol{F}_{\text {reg. }}$.

The result of the regression analysis, $\boldsymbol{F}_{\text {reg }}$, is almost same as the measurement error, $\boldsymbol{F}_{\text {diff. }}$ From this result, the corrected force, $\boldsymbol{F}_{\text {corrected }}$, is calculated using the following equation:

$$
\boldsymbol{F}_{\text {corrected }}=\boldsymbol{F}_{\text {trans }}-\boldsymbol{F}_{\text {reg }}
$$

Figure 6 shows the root mean square (RMS) values of the dynamic errors, $\boldsymbol{F}_{\text {diff }}$, and that of the residual errors after dynamic correction, $\boldsymbol{F}_{\text {res }}$ :

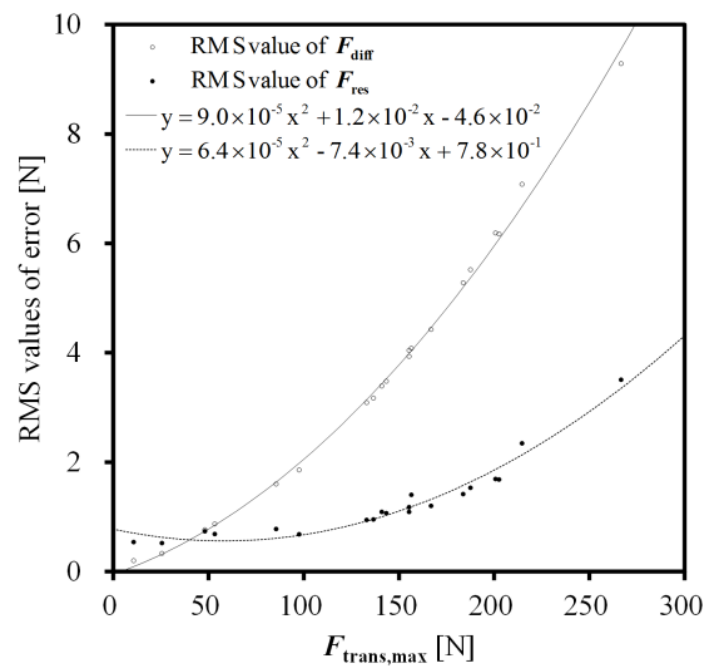

Fig. 6. RMS values of the dynamic errors, $\boldsymbol{F}_{\text {diff }}=\boldsymbol{F}_{\text {trans }}$ - $\boldsymbol{F}_{\text {mass, }}$ and RMS values of the residual errors of the corrected values, $\boldsymbol{F}_{\text {res }}=\boldsymbol{F}_{\text {corrected }}-\boldsymbol{F}_{\text {mass }}$.

$$
\boldsymbol{F}_{\text {res }}=\boldsymbol{F}_{\text {corrected }}-\boldsymbol{F}_{\text {mass }}
$$

The RMS values of $\boldsymbol{F}_{\text {res }}$ are effectively smaller than that of $\boldsymbol{F}_{\text {diff. }}$ The dynamic error correction parameter, $\boldsymbol{C}_{\mathbf{d}}$, gives satisfactory results.

The RMS values of $\boldsymbol{F}_{\text {diff }}$ and $\boldsymbol{F}_{\text {res }}$ look almost quadratic functions of the maximum force, $\boldsymbol{F}_{\text {trans,max }}$. The solid and dashed curves in Fig. 5 are regression curve of the RMS values of $\boldsymbol{F}_{\text {diff }}$ and $\boldsymbol{F}_{\text {res }}$ respectively. The RMS value of the differences between RMS value of $\boldsymbol{F}_{\text {diff }}$ and its regression curve and that between RMS value of $\boldsymbol{F}_{\text {res }}$ and its regression curve are $1.37 \times 10^{-1} \mathrm{~N}$ and $1.36 \times 10^{-1} \mathrm{~N}$ respectively. This results show that the RMS values of $\boldsymbol{F}_{\text {diff }}$ and $\boldsymbol{F}_{\text {res }}$ could be estimated from $\boldsymbol{F}_{\text {trans,max }}$

\section{Estimation of Uncertainty}

The standard uncertainty, $\boldsymbol{u}_{\text {trans}}$, of the un-corrected force, $\boldsymbol{F}_{\text {trans }}$, is estimated as follows:

$$
\boldsymbol{u}_{\text {trans }}=\sqrt{\boldsymbol{u}_{\text {diff }}^{2}+\boldsymbol{u}_{\mathrm{LMM}}^{2}}
$$

where $\boldsymbol{u}_{\text {diff }}$ is the RMS values of $\boldsymbol{F}_{\text {diff }}$ during the impact shown in Fig. 6. $\boldsymbol{u}_{\text {LMM }}$ in the experiment is estimated to be $1.2 \times 10^{-4} \mathrm{~N}$ and is negligible ${ }^{(5)}$. As shown in Fig. 6, it is possible that $\boldsymbol{u}_{\text {diff }}$ is assumed to be a function of the square of the peak value of the force, $\boldsymbol{F}_{\text {trans,max }}$, as follows:

$$
\boldsymbol{u}_{\text {trans }}=\boldsymbol{E}_{\mathrm{S}, \mathbf{1}} \cdot \boldsymbol{F}_{\text {transmax }}^{2}+\boldsymbol{E}_{\mathrm{S}, 2} \cdot \boldsymbol{F}_{\text {transmax }}+\boldsymbol{E}_{\mathrm{S}, 3}
$$

where the constants $\boldsymbol{E}_{\mathbf{S}, \mathbf{1}}, \boldsymbol{E}_{\mathbf{S}, \mathbf{2}}$ and $\boldsymbol{E}_{\mathbf{S}, \mathbf{3}}$ are parameters for estimating the dynamic error of the un-corrected force, $\boldsymbol{F}_{\text {trans, }}$ which is calculated based on conventional static calibration.

Just like the $\boldsymbol{u}_{\text {trans }}$, the standard uncertainty, $\boldsymbol{u}_{\text {corrected }}$, of the corrected force, $\boldsymbol{F}_{\text {corrected }}$, is estimated as follows:

$$
\boldsymbol{u}_{\text {corrected }}=\sqrt{\boldsymbol{u}_{\mathrm{res}}^{2}+\boldsymbol{u}_{\mathrm{LMM}}^{2}}
$$

where $\boldsymbol{u}_{\text {res }}$ is the RMS values of the residual error, $\boldsymbol{F}_{\text {res }}$, during the impact shown in Fig. 6. It is possible that $\boldsymbol{u}_{\text {res }}$ is assumed to be a function of the square of the peak value of the force, $\boldsymbol{F}_{\text {trans,max }}$, as follows:

$$
\boldsymbol{u}_{\text {corrected }}=\boldsymbol{E}_{\mathrm{D}, \mathbf{1}} \cdot \boldsymbol{F}_{\text {tranşmax }}^{2}+\boldsymbol{E}_{\mathrm{D}, 2} \cdot \boldsymbol{F}_{\text {tranșmax }}+\boldsymbol{E}_{\mathrm{D}, \mathbf{3}}
$$

where the constants $\boldsymbol{E}_{\mathbf{D}, \mathbf{1}}, \boldsymbol{E}_{\mathbf{D}, \mathbf{2}}$ and $\boldsymbol{E}_{\mathbf{D}, \mathbf{3}}$ are parameters for estimating the residual error of the corrected force, $\boldsymbol{F}_{\text {corrected }}$, which is estimated by the dynamic error correction method that has been proposed.

\section{Correction Software}

From the above experiments, software for correcting 
the dynamic error in the impact force measurement of the force sensor has been developed. It works on Microsoft Windows environment. Figure 7 shows a screenshot of the software.

This software processes the output waveform from the force sensor, $\boldsymbol{V}_{\text {trans }}$, into [a] waveform of force measured by the sensor, $\boldsymbol{F}_{\text {trans }}$, which is based on static calibration result, [b] waveform of dynamic error, $\boldsymbol{F}_{\text {reg, }}$ [c] waveform of corrected force, $\boldsymbol{F}_{\text {corrected }}$. Furthermore, this software calculates [d] the standard uncertainty, $\boldsymbol{u}_{\text {trans, of the }}$ un-corrected force, $\boldsymbol{F}_{\text {trans, }}$, and [e] the standard uncertainty, $\boldsymbol{u}_{\text {corrected }}$, of the corrected force, $\boldsymbol{F}_{\text {corrected }}$.

The software shows them to monitor window and also outputs them to an output file. The format of the input waveform file is a text format which is described the sampling interval in time and the sequence of voltage indication of rated output of the sensor in mili bolt per bolt. The format of the output data file is a CSV format. The input and output data are as follows,

[A] Input data which is manually inputted from the operational window.

[1] Name of input text-file which contains the data set of the voltage indication of rated output of the sensor, $\boldsymbol{V}_{\text {trans }}(\mathrm{i})(0 \leq \mathrm{i}<\mathrm{N}-1)$

[2] Static calibration coefficient: $\boldsymbol{C}_{\mathbf{s}}$

[3] Parameter for dynamic correction: $\boldsymbol{C}_{\mathbf{d}}$

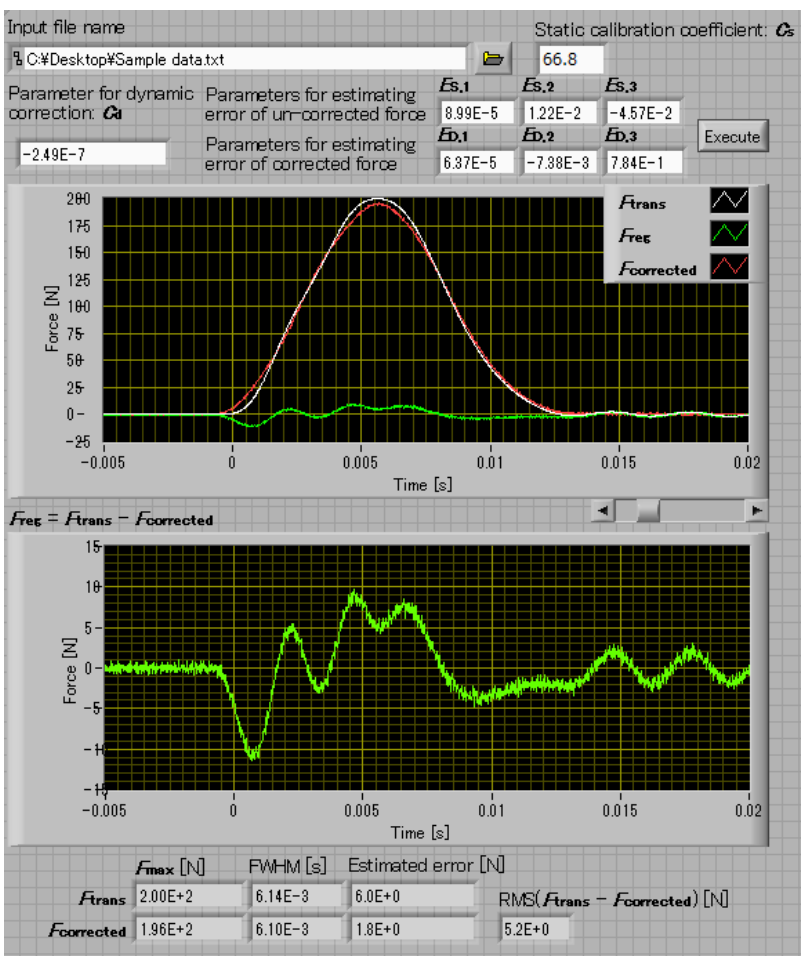

Fig. 7. Screenshot of the correction software.
[4] Parameters for estimating error of the un-corrected force, $\boldsymbol{F}_{\text {trans }}$, which is calculated based on conventional static calibration: $\boldsymbol{E}_{\mathrm{S}, \mathbf{1}}, \boldsymbol{E}_{\mathrm{S}, \mathbf{2}}$ and $\boldsymbol{E}_{\mathrm{S}, \mathbf{3}}$.

[5] Parameters for estimating error of the corrected force, $\boldsymbol{F}_{\text {corrected }}$, which is estimated by the dynamic error correction method: $\boldsymbol{E}_{\mathrm{D}, \mathbf{1}}, \boldsymbol{E}_{\mathrm{D}, \mathbf{2}}$ and $\boldsymbol{E}_{\mathrm{D}, \mathbf{3}}$.

[B] Output data which is shown in the display and written in a CSV-file.

[1] Waveform of $\boldsymbol{F}_{\text {trans }}, \boldsymbol{F}_{\text {reg }}$ and $\boldsymbol{F}_{\text {corrected }}$

[2] Parameters for $\boldsymbol{F}_{\text {corrected }}$ the maximum value,

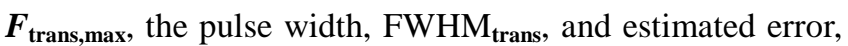
$u_{\text {trans }}$

[3] Parameters for $\boldsymbol{F}_{\text {corrected }}$ the maximum value, $\boldsymbol{F}_{\text {corrected,max }}$, the pulse width, FWHM corrected, and estimated error, $\boldsymbol{u}_{\text {corrected }}$.

[4] RMS value of the difference between $\boldsymbol{F}_{\text {trans }}$ and $\boldsymbol{F}_{\text {corrected }}$

\section{Conclusions}

The effectiveness of proposed method for correcting dynamic error in the impact force measurement of force sensor is experimentally evaluated. On the basis of experimental result, software which corrects the dynamic error using the output signal of the sensor itself has been developed. The software has been modified to load input text-file which contains $\boldsymbol{V}_{\text {trans }}$ instead of conventional input text-file which contains $\boldsymbol{F}_{\text {trans }}$.

\section{Acknowledgment}

This work was supported in part by a research-aid fund of the NSK Foundation for the Advancement of Mechatronics (NSK-FAM) and the Grant-in-Aid for Scientific Research (B) 24360156 (KAKENHI 24360156).

\section{References}

(1) Y. Fujii : "Toward Establishing Dynamic Calibration Method for Force Transducers", IEEE Transactions on Instrumentation and Measurement, Vol. 58, pp. 2358-2364, 2009

(2) Y. Fujii : "Measurement of the electrical and mechanical responses of a force transducer against impact forces", Review of Scientific Instruments, Vol. 77, 085108, 2006

(3) Y. Fujii and K. Maru : "Self-correction method for 
dynamic measurement error of force sensors", Experimental Techniques, Vol. 35, pp. 15-20, 2011

(4) N. Miyashita, K. Watanabe, K. Irisa, H. Iwashita, R. Araki, A. Takita, T. Yamaguchi and Y. Fujii : "Software for Correcting the Dynamic Error of Force Transducers", Sensors, Vol. 14, pp. 12093-12103, 2014

(5) Y. Fujii : "Frictional characteristics of an aerostatic linear bearing", Tribology International, Vol. 39, pp. 888-896, 2006 